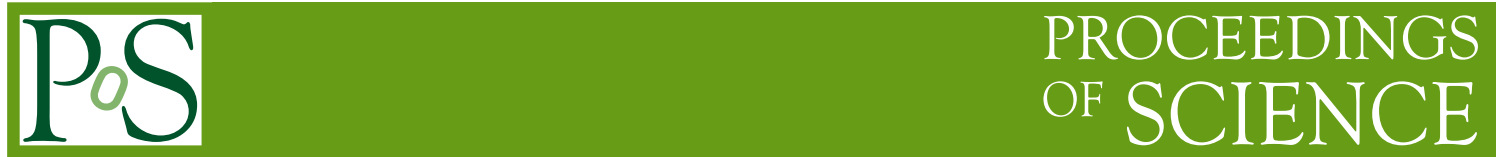

\title{
Model independent results for nucleon structure
}

\author{
Gerald A. MILLER ${ }^{* \dagger}$ \\ University of Washington, USA \\ E-mail: miller@phys.washington.edu
}

I review recent results regarding the nucleon charge and magnetization densities as well as the shape of the nucleon. First, some phenomenolgical considerations that show that the shape of the proton is not round are discussed. Then model independent results regarding the neutron and proton charge density, and the proton magnetization density are presented. Finally, I show how the spin-dependent densities that reveal the shape of the proton can be measured via their relation with transverse momentum distributions. The present work is made possible by the recent tremendous experimental progress made at Bates, Mainz and Jefferson Laboratory.

LIGHT CONE 2008 Relativistic Nuclear and Particle Physics

July 7-11, 2008

Mulhouse, France

\footnotetext{
* Speaker.

${ }^{\dagger}$ This work is partially supported by the USDOE.
} 


\section{Phenomenology}

The electromagnetic form factors are matrix elements of the current operator, $J^{\mu}(x)$, between nucleon states of different momentum:

$$
\left\langle p^{\prime}, \lambda^{\prime}\left|J^{\mu}(0)\right| p, \lambda\right\rangle=\bar{u}\left(p^{\prime}, \lambda^{\prime}\right)\left(\gamma^{\mu} F_{1}\left(Q^{2}\right)+i \frac{\sigma^{\mu \alpha}}{2 M} q_{\alpha} F_{2}\left(Q^{2}\right)\right) u(p, \lambda)
$$

where $q_{\alpha}=p_{\alpha}^{\prime}-p_{\alpha}, Q^{2} \equiv-q^{2}>0, M$ is the nucleon mass and the light-cone helicities are $\lambda, \lambda^{\prime}$. The Sachs form factors are: $G_{E}\left(Q^{2}\right) \equiv F_{1}\left(Q^{2}\right)-\frac{Q^{2}}{4 M^{2}} F_{2}\left(Q^{2}\right), G_{M}\left(Q^{2}\right) \equiv F_{1}\left(Q^{2}\right)+F_{2}\left(Q^{2}\right)$. The early expectation, based on a simple application of helicity conservation at very high values of the momentum transfer, was that the $Q F_{2}\left(Q^{2}\right) / 2 M F_{1} \sim \frac{m_{\text {quark }}}{Q} \rightarrow \frac{G_{E}\left(Q^{2}\right)}{G_{M}\left(Q^{2}\right)}=$ const, which is also obtained from non-relativistic considerations. Thus the expectation was that, at sufficiently large momentum transfer, $G_{E} / G_{M}$ would be flat and the $Q F_{2} / F_{1}$ would fall. However, the reverse was true [1].

It is necessary to comment on the meaning of these form factors. In the Breit frame, with $\mathbf{p}=-\mathbf{p}^{\prime}, G_{E}$ is the nucleon helicity flip matrix element of $J^{0}$. However, any probability or density interpretation of $G_{E}$ is spoiled by a non-zero value of $Q^{2}$, no matter how small. The initial and final states have different momentum, and therefore relativistically have different wave functions. Any attempt to analytically correct for the total momentum by incorporating relativistic corrections in a $p^{2} / m_{q}^{2}$ type of expansion is doomed by the presence of the very light current quark mass, $m_{q}$. That is, at small values of $Q^{2}$, one finds

$$
G_{E}^{n} \sim Q^{2}\left(\int d^{3} r\left(r^{2}|\psi|^{2}+\frac{C}{m_{q}^{2}}\right),\right.
$$

where the first term represents the expected effect depending on the square of the wave function and the term $C$ represents the unknown boost correction.

So to analyze form factors using a model, the model must be relativistic. We chose [2] to use a relativistic model using light front coordinates. These useful coordinates involve the use of a "time" $x^{+}=(c t+z) / \sqrt{2}=\left(x^{0}+x^{3}\right) / \sqrt{2}$. The corresponding evolution operator is the not the Hamiltonian, $p^{0}$, but instead $p^{-}=\left(p^{0}-p^{3}\right) / \sqrt{2}$. The orthogonal spatial coordinate is $x^{-}=$ $\left(x^{0}-x^{3}\right) / \sqrt{2}$. If one quantizes at $x^{+}=0$, then $x^{-}=\sqrt{2} z$, and this is why $x^{-}$is thought of as the spatial variable. The canonically conjugate momentum is given by $p^{+}=\left(p^{0}+p^{3}\right) / \sqrt{2}$. We note that $p_{\mu} x^{\mu}=p^{-} x^{+}+p^{+} x^{-}-\mathbf{p} \cdot \mathbf{b}$. The transverse coordinates perpendicular to the 0 and 3 directions are denoted as $\mathbf{b}$ and $\mathbf{p}$. Using these variables allows the separation of center of mass and relative position variables in a manner similar to that of the usual non-relativistic treatment. The key feature is that transverse boosts act like the non-relatistic boosts.

We used these variables to formulate a Poincare invariant, relativistic constituent quark model in 1995 and predict the qualitative behavior of form factors measured five years later. Please see Figs. 10, 11 of Ref. [2]. More detailed analysis [3]showed that the flat nature of $\frac{Q F_{2}}{F_{1}}$ results from orbital angular momentum of the quarks inherent in the lower component of quark Dirac spinors.

So we had a reasonable model, which includes quark orbital angular momentum. I was faced with the challenge of relating orbital angular momentum to a potential non-spherical shape of the proton. The notion that the proton might not be a sphere has its impetus in the discovery that the spins of quarks and anti-quarks account for only about $30 \%$ of the total angular momentum. It 
seems natural to associate non-zero orbital angular momentum with a non-spherical shape, but the Wigner-Eckart theorem says that the proton has no quadrupole moment. In response, I introduced spin-dependent densities SDD, and later learned these are common in condensed matter physics.

Spin-dependent density operators We interpret orbital angular momentum in terms of the shapes of the proton exhibited through the rest-frame ground-state matrix elements of spin-dependent density operators [4]. The density operator is $\widehat{\rho}(\mathbf{r})=\sum_{i} \delta\left(\mathbf{r}-\mathbf{r}_{i}\right)$, where $\mathbf{r}_{i}$ is the position operator of the $i$ th particle. For particles of spin $1 / 2$ one can measure the combined probability that a particle is at a given position $\mathbf{r}$ and has a spin in an arbitrary, fixed direction specified by a unit vector $\mathbf{n}$. The coordinate-space spin-dependent density SDD operator is $\widehat{\rho}(\mathbf{r}, \mathbf{n})=\sum_{i} \delta\left(\mathbf{r}-\mathbf{r}_{i}\right) \frac{1}{2}\left(1+\sigma_{i} \cdot \mathbf{n}\right)$.

To understand the connection between the spin-dependent density and orbital angular momentum, consider a first example of a single charged particle moving in a fixed, rotationallyinvariant potential in an energy eigenstate $\left|\Psi_{1,1 / 2, s}\right\rangle$ of quantum numbers: $l=1, j=1 / 2$, polarized in the direction $\widehat{\mathbf{s}}$ and radial wave function $R\left(r_{p}\right)$. The wave function can be written as $\left(\mathbf{r}_{p}\left|\Psi_{1,1 / 2, s}\right\rangle=R\left(r_{p}\right) \sigma \cdot \hat{\mathbf{r}}_{p}|s\rangle\right.$. The ordinary density $\rho(r)=\left\langle\Psi_{1,1 / 2, s}\left|\boldsymbol{\delta}\left(\mathbf{r}-\mathbf{r}_{p}\right)\right| \Psi_{1,1 / 2, s}\right\rangle=R^{2}(r)$, a spherically symmetric result because the effects of the Pauli spin operator square to unity. But the matrix element of the SDD is more interesting:

$$
\rho(\mathbf{r}, \mathbf{n})=\frac{R^{2}(r)}{2}\langle\hat{s}|\sigma \cdot \hat{\mathbf{r}}(1+\sigma \cdot \hat{\mathbf{n}}) \sigma \cdot \hat{\mathbf{r}}| \widehat{s}\rangle .
$$

The magnetic quantum defines an axis, $\mathbf{s}$ and the direction of vectors can be represented in terms of this axis: $\hat{\mathbf{s}} \cdot \hat{\mathbf{r}}=\cos \theta$. Suppose $\hat{\mathbf{n}}$ is either parallel or anti-parallel to the direction of the proton angular momentum vector $\hat{\mathbf{s}}$. Then $\rho(\mathbf{r}, \mathbf{n}=\hat{\mathbf{s}})=R^{2}(r) \cos ^{2} \theta, \rho(\mathbf{r}, \mathbf{n}=-\hat{\mathbf{s}})=R^{2}(r) \sin ^{2} \theta$, and the non-spherical shape is exhibited. The average of these two cases is a spherical shape.

We computed momentum-space SDDs [4] using our model[2] model and obtained a variety of unusual shapes. See Figs. 2,3 of [4]. A possible shape of the proton is shown here in Fig. 1.

The logic is that using a model wave function, in rough agreement with data for form factors, leads to a non-spherical shape of the SDD. However, there was no direct connection between experiment and the shapes. We shall return to issue after discussing other model independent results for charge and magnetization densities.

\section{Model independent neutron charge density}

The neutron has no net charge, but the charge density need not vanish. So we can ask, "Is the central charge density negative or positive?". Long-standing existing answers are based on models For example,. the neutron can make a spontaneous quantum transition to a $p \pi^{-}$state [5]. The light pion spreads out over a larger region of space than the proton to cause a negative charge density at the edge of the neutron and positive one at the center. A similar result is obtained with the different one-gluon exchange force, acting repulsively between two negatively charged d-quarks. But enough about models!

The starting point for a model independent analysis is the use of transversely localized nucleon states $[6,7,8]$ :

$$
\left|p^{+}, \mathbf{R}=\mathbf{0}, \lambda\right\rangle \equiv \mathscr{N} \int \frac{d^{2} \mathbf{p}}{(2 \pi)^{2}}\left|p^{+}, \mathbf{p}, \lambda\right\rangle
$$




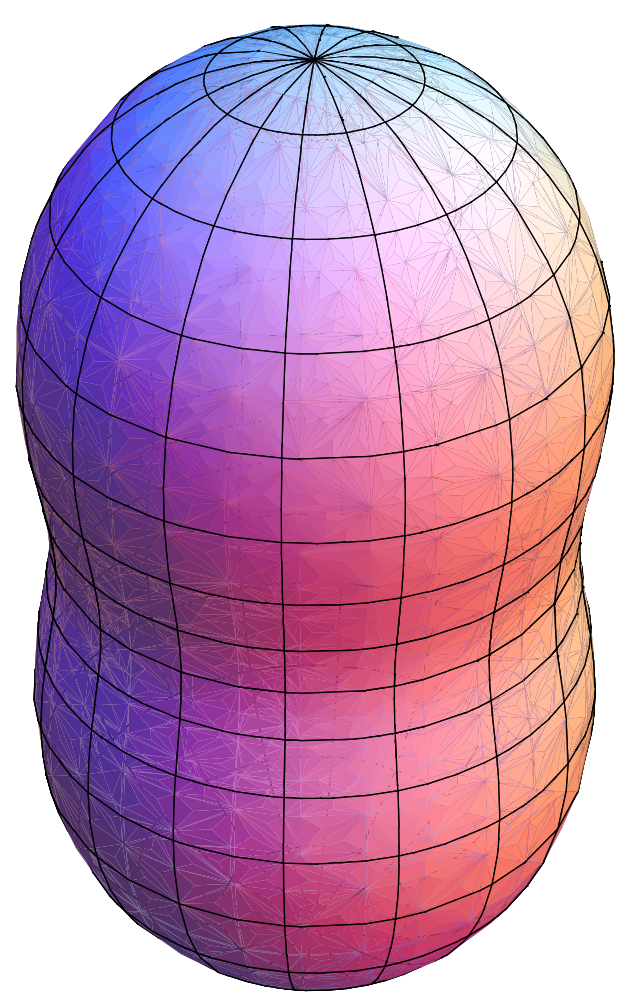

Figure 1: Possible shape of the proton. The angular momentum of the proton is up, as is the spin of the struck quark. The probability for a quark to have a momentum direction at a given angle is proportional to the distance from the center. In this case, a high momentum quark of spin up is twice as likely to have its momentum parallel to its spin as it is to have be perpendicular.

where $\left|p^{+}, \mathbf{p}, \lambda\right\rangle$ are light-cone helicity eigenstates and $\mathscr{N}$ is a normalization factor. The range of integration in Eq. (2.1) must be restricted to $|\mathbf{p}| \ll p^{+}$to maintain the interpretation of a nucleon moving with well-defined longitudinal momentum[7]. Thus we use the infinite momentum frame.

Using Eq. (2.1) sets the transverse center of momentum of a state of total very large momentum $p^{+}$to zero, so that transverse distance $\mathbf{b}$ relative to $\mathbf{R}$. can be defined. Thus one defines a useful combination of quark-field operators [7]:

$$
\hat{O}_{q}(x, \mathbf{b}) \equiv \int \frac{d x^{-}}{4 \pi} q_{+}^{\dagger}\left(-\frac{x^{-}}{2}, \mathbf{b}\right) q_{+}\left(\frac{x^{-}}{2}, \mathbf{b}\right) e^{i x p^{+} x^{-}}
$$

where the subscript + denotes the use of independent quark field operators. The impact parameter 
dependent PDF is defined [7] as:

$$
q(x, \mathbf{b}) \equiv\left\langle p^{+}, \mathbf{R}=\mathbf{0}, \lambda\left|\hat{O}_{q}(x, \mathbf{b})\right| p^{+}, \mathbf{R}=\mathbf{0}, \lambda\right\rangle .
$$

This is the basic density that can be obtained, giving the probablitiy of finding a quark at a transverse position that carries a fraction $x$ of the the protons plus component of momentum. The use of Eq. (2.1) in Eq. (2.3) allows one to show [9] that $q(x, \mathbf{b})$ is the two-dimensional Fourier transform of the GPD $H_{q}$ :

$$
q(x, \mathbf{b})=\int \frac{d^{2} q}{(2 \pi)^{2}} e^{i \mathbf{q} \cdot \mathbf{b}} H_{q}\left(\xi=0, x, t=-\mathbf{q}^{2}\right),
$$

One obtains a relation between $q(x, \mathbf{b})$ and the form factor [6] by integrating $q(x, \mathbf{b})$ over all values of $x$. This sets the value of $x^{-}$to 0 , so that a density appears in the matrix element. If one also multiplies by the quark charge $e_{q}$ (in units of $e$ ), sums over quark flavors, and uses the sum rule relating the GPD to the form factor, the resulting infinite-momentum-frame IMF parton charge density in transverse space is

$$
\rho(b) \equiv \sum_{q} e_{q} \int d x q(x, \mathbf{b})=\int \frac{d^{2} q}{(2 \pi)^{2}} F_{1}\left(Q^{2}=\mathbf{q}^{2}\right) e^{i \mathbf{q} \cdot \mathbf{b}} .
$$

We exploit Eq. (2.5) by using using recent parameterizations [10,11] of measured form factors to determine $\rho(b)$. The charge densities of the proton and neutron are shown in Fig. 1 of [9]. The surprising feature is the negative central value of the neutron charge density. This results from the negative definite nature of $F_{1}$ [9]. The neutron charge density has interesting features, as shown in Fig. 2 of [9] which displays the quantity $b \rho(b)$. It is the integral of this quantity that integrates to 0 . The neutron charge density is negative at the center, positive in the middle, and again negative at the outer edge. The medium-ranged positive charge density is sandwiched by inner and outer regions of negative charge. This interesting behavior needs to be better understood.

One gains information about individual $u$ and $d$ quark densities by invoking charge symmetry [12] so that $u, d$ densities in the proton are the same as $d, u$ densities in the neutron. The results, [9] are that the central up quark density is larger than that of the down quark by about $30 \%$.

Proton Magnetization Density We recently showed [13], that the two-dimensional Fourier transform of the Pauli form factor $F_{2}$ plays the role of the magnetization density.

\section{Measuring the Non-Spherical Shape of the Nucleon}

While the matrix elements of the spin-density operator reveal highly non-spherical densities, experimentally determining the proton's non-spherical nature has remained a challenge. Here we explain how matrix elements of the spin-dependent density may be measured using their close connection with transverse momentum dependent parton densities [14].

The field-theoretic version of the spin-dependent charge density operator is a generalization of the operator defined in Ref. [4]:

$$
\widehat{\rho}_{\mathrm{REL}}(\mathbf{K}, \mathbf{n})=\left.\int \frac{d^{3} \xi}{(2 \pi)^{3}} e^{-i \mathbf{K} \cdot \xi} \bar{\psi}(0) \gamma^{0}\left(1+\gamma \cdot \mathbf{n} \gamma_{5}\right) \mathscr{L}(0, \xi ; \text { path }) \psi(\xi)\right|_{t=\xi^{0}=0},
$$


where $\psi$ is a quark field operator and flavor indices are omitted. The quark field operators are evaluated at equal time and accompanied by a path-ordered exponential link operator $\mathscr{L}((0, \xi$; path $)$ needed for color-gauge invariance. This matrix element gives the probability of finding a quark of three momentum $\mathbf{K}$ and spin direction $\mathbf{n}$. It depends on the direction of three vectors $\mathbf{K}, \mathbf{n}$ and the direction of the spin polarization. Compared with the orginally introduced SDD, there is an extra $\gamma^{0}$ in front of the term that depends on $\mathbf{n}$.

Measuring the spin-dependent density requires that the system be probed with identical initial and final states. But this condition also enters in measurements of both ordinary and transversemomentum-dependent TMD parton distributions. The latter [15] are:

$$
\Phi^{[\Gamma]}(x, \mathbf{K})=\left.\int \frac{d \xi^{-} d^{2} \xi_{T}}{2(2 \pi)^{3}} e^{i K \cdot \xi}\left\langle P, S\left|\bar{\psi}(0) \Gamma \mathscr{L}\left(0, \xi ; n_{-}\right) \psi(\xi)\right| P, S\right\rangle\right|_{\xi^{+}=0},
$$

where the specific path $n_{-}$is that of their Appendix B. The functions $\Phi^{[\Gamma]}$ depend on the fractional momentum $x=K^{+} / P^{+}$, the trasnverse momentum $\mathbf{K}$, and the proton spin direction. The operator $\Gamma$ can be any Dirac operator. In particular, the shape of the proton is revealed [14] through

$$
\Phi^{\left[i \sigma^{i+} \gamma_{5}\right]}(x, \mathbf{K})=S_{T}^{i} h_{1}\left(x, K^{2}\right)+\frac{K^{i} K^{j}-\frac{1}{2} \delta_{i j}}{M^{2}} h_{1 T}^{\perp}\left(x, K^{2}\right) .
$$

It is therefore tempting to try to associate an SDD such as that of Eq. (3.1) with TMDs, but one difference is essential. Parton density operators Eq. (3.2) depend on quark-field operators defined at a fixed light cone time $\xi^{+}=\xi^{3}+\xi^{0}=0$ while our SDD is an equal-time, $\xi^{0}=0$, correlation function. However, a relation between the two sets of operators is obtained [14] by integrating the TMD over all values of $x$ setting $\xi^{-}$to zero, and integrating Eq. (3.1) over all values of $K_{z}$ so that $\xi^{3}=0$. After integration, $\xi^{ \pm}=0$ for both functions. Computed models for the transverse spin dependent densities are shown in Figs. 1,2 of [14].

The term $h_{1 T}^{\perp}$ causes distinctive experimental signatures in semi-inclusive leptoproduction hadron production experiments see the list of references in [14]. In each of these cases, the momentum of the virtual photon and its vector nature provide the analogue of the vector $\mathbf{n}$ needed to define the spin-dependent density. The hadronic transverse momentum provides the third, $\mathbf{K}_{T}$.

Of special interest is the reaction $e p \uparrow \rightarrow e^{\prime} \pi X$ Here the term $h_{1 T}^{\perp}$ causes a distinctive oscillatory dependence on the angle $3 \phi-\phi_{S_{1}}$, [16] where $\phi$ is the angle between the momentum of the outgoing lepton and the reaction plane in the lepton center of mass frame, and $\phi_{S_{1}}$ denotes the direction of polarization with respect to the reaction plane.

It is very exciting that experiments planned at Jefferson Laboratory aim to specifically measure $h_{1 T}^{\perp}$ [17] and therefore determine whether or not the proton is round. We also note that the non-spherical shape of the nucleon has been established in lattice QCD by computing appropriate moments of impact parameter dependent gpds. See the talk of Zanotti in this workshop.

Our summary of SDDs is that these are closely related to TMDs. If $h_{1 T}^{\perp}$ is not 0 , the proton is not round. Experiment can show that the proton is not round.

\section{References}

[1] M. K. Jones et al. "G(E(p))/G(M(p)) ratio by polarization transfer in e(pol.) $\mathrm{p} \rightarrow \mathrm{e}$ (pol.)," Phys. Rev. Lett. 84 (2000) 1398. 
[2] M. R. Frank, B. K. Jennings and G. A. Miller, "The Role of Color Neutrality in Nuclear Physics-Modifications of Nucleonic Wave Functions," Phys. Rev. C 54 (1996) 920.

[3] G. A. Miller and M. R. Frank, " $\mathrm{Q} 2$ independence of $\mathrm{QF}_{2} / F_{1}$, Poincare invariance and the non-conservation of helicity”, Phys. Rev. C 65 (2002) 065205.

[4] G. A. Miller, “Shapes of the proton,” Phys. Rev. C 68, (2003) 022201 (R).

[5] A. W. Thomas, S. Théberge, and G. A. Miller, “The Cloudy Bag Model Of The Nucleon,” Phys. Rev. D24 216 (1981) ; G. A. Miller, "Light front cloudy bag model: Nucleon electromagnetic form factors," Phys. Rev. C 66, (2002) 032201.

[6] D. E. Soper, "The Parton Model And The Bethe-Salpeter Wave Function," Phys. Rev. D 151 (1977) 114.

[7] M. Burkardt, "Impact parameter space interpretation for generalized parton distributions," Int. J. Mod. Phys. A 18 (2003) 173. "Impact parameter dependent parton distributions and off-forward parton distributions for $\zeta \rightarrow 0$,” Phys. Rev. D 62 (2000) 071503 [Erratum-ibid. D 66 (2002) 119903].

[8] M. Diehl, “Generalized parton distributions in impact parameter space," Eur. Phys. J. C 25 (2002) 223 [Erratum-ibid. C 31 (2003) 277].

[9] G. A. Miller, “Charge Density of the Neutron,” Phys. Rev. Lett. 99 (2007) 112001.

[10] R. Bradford, A. Bodek, H. Budd and J. Arrington, "A new parameterization of the nucleon elastic form factors," Nucl. Phys. Proc. Suppl. 159(2006) 127.

[11] J. J. Kelly, "Simple parametrization of nucleon form factors," Phys. Rev. C 70 (2004) 068202.

[12] G. A. Miller, B. M. K. Nefkens and I. Slaus, "Charge Symmetry, Quarks And Mesons," Phys. Rept. 194 (1990) 1.

[13] G. A. Miller, E. Piasetzky and G. Ron, "Proton Electromagnetic Form Factor Ratios at Low $Q^{2}$,", to be published in PRL, arXiv:0711.0972 [nucl-th].

[14] G. A. Miller, "Densities, Parton Distributions, and Measuring the Non-Spherical Shape of the Nucleon," Phys. Rev. C 76 (2007) 065209.

[15] P. J. Mulders and R. D. Tangerman, "The complete tree-level result up to order 1/Q for polarized deep-inelastic leptoproduction,” Nucl. Phys. B 461 (1996) 197, [Erratum-ibid. B 484 (1997) 538].

[16] D. Boer and P. J. Mulders, “Time-reversal odd distribution functions in leptoproduction," Phys. Rev. D 57 (1998) 5780.

[17] H. Avakian, et al. "Transverse Polarization Effects in Hard Scattering at CLAS12 Jefferson Laboratory", LOI12-06-108, and private communication. 